\title{
The Origin and Structure of the Magnetic Fields and Currents of AGN Jets
}

\author{
Denise Gabuzda \\ Physics Department, University College Cork, Cork, Ireland; d.gabuzda@ucc.ie \\ Academic Editors: Jose L. Gómez, Alan P. Marscher and Svetlana G. Jorstad \\ Received: 17 October 2016; Accepted: 3 January 2017; Published: 2 February 2017
}

\begin{abstract}
This paper reviews observational evidence obtained to date about the overall structure of the magnetic fields in the jets of Active Galactic Nuclei (AGN). Because they are sensitive to the line-of-sight magnetic-field component, Faraday rotation observations of AGN jets provide an effective tool for searching for toroidal jet magnetic fields, whose line-of-sight component changes systematically across the jet. Transverse Faraday rotation measure (RM) gradients providing direct evidence for helical/toroidal magnetic fields have been reliably detected in nearly 40 AGN on parsec scales. Helical magnetic fields are believed to form due to the combined action of the rotation of the central black hole and accretion disk, and these observations demonstrate that at least some of this helical field survives to distances well beyond the Very Long Baseline Interferometry (VLBI) core. Observations of reversals in the direction of the transverse RM gradients in a number of AGN provide evidence for a "return" magnetic field forming a nested helical-field structure with oppositely directed azimuthal components in the inner and outer regions of the helical magnetic field. The collected data now provide firm evidence for a predominance of inward jet currents on parsec scales and outward currents on scales greater than a few tens of parsecs. This suggests a global pattern of magnetic fields and currents with an inward current near the jet axis and an outward current farther from the jet axis, with these currents closing in the accretion disk and far out in the radio lobes, forming a self-consistent set of fields and currents together with the implied nested helical-field structure.
\end{abstract}

Keywords: active galactic nuclei; relativistic jets; magnetic fields; radio interferometry

\section{Introduction}

The central regions of powerful Active Galactic Nuclei (AGN) radiate huge amounts of energy; the strongest AGN are about $10^{5}$ times more luminous than an entire normal galaxy such as the Milky Way. The source of this phenomenal energy is believed to be the gravitational energy released by matter accreting onto a supermassive ( $\sim 10^{9}$ times the mass of the Sun) black hole at the galactic centre.

About $10-15 \%$ or so of all AGN are "radio-loud" (ratio of its radio $(5 \mathrm{GHz})$ to its optical (B-band) flux $\geq 10$; [1]) Radio emission is predominantly associated with jets ejected from the central region of the AGN, presumably along the rotational axis of the central supermassive black hole, and the lobes that they inflate. This radio emission is synchrotron radiation emitted by relativistic electrons moving through regions with magnetic (B) field; the jets presumably create conditions where electrons can be accelerated to high energies and magnetic fields can also be generated and amplified.

The jets are present on the smallest scales that can be probed with Very Long Baseline Interferometry (VLBI), and sometimes extend out to scales of many kiloparsecs. The synchrotron radiation given off by each individual electron is highly concentrated in the direction of motion of the electron, and the radiation observed for an ensemble of relativistic electrons will, in general, be linearly polarized if the synchrotron $\mathbf{B}$ field is at least partially ordered. Linear-polarization observations can thus provide direct information about the degree of order and orientation of the $\mathbf{B}$ field giving rise to the observed synchrotron radiation, and thus play a key role in studies of the conditions in and around 
AGN jets. The maximum theoretical degree of polarization for the synchrotron radiation associated with AGN jets is usually taken to be about $75 \%$, with the degree of polarization decreasing if the local B field is not completely ordered, and with the observed polarization direction being perpendicular to the synchrotron B field in optically thin regions. High-resolution multi-wavelength observations can provide valuable information about the spectrum of the radio emission and the possible presence of Faraday rotation arising at various locations between the emission region and the observer.

\section{Faraday Rotation Gradients and Helical/Toroidal B Fields}

Faraday rotation is a rotation of the plane of polarization of an electromagnetic wave that occurs when it passes through a region with free charges and magnetic field. Any electromagnetic wave can be described as the sum of any two orthogonal components, usually considered in radio astronomy to be right circularly polarized (RCP) and left circularly polarized (LCP). Due to asymmetry in the interactions between the local free charges and the RCP and LCP components of the polarized wave, these two components have different indices of refraction, and therefore different speeds of propagation through the magnetized medium.

When the wave propagates through a vacuum, the electric-field vectors for these two components rotate in opposite directions at the same rate, preserving the orientation of the plane of linear polarization, $\chi$. When the wave propagates through a magnetized plasma, the difference in the speeds of the RCP and LCP waves induces a delay between these components, manifesting as a rotation in the plane of polarization. The amount of rotation depends on the strength of the ambient magnetic field $\mathbf{B}$, the number density of charges in the plasma $n_{e}$, the charge $e$ and mass $m$ of these charges, and the wavelength of the radiation $\lambda$ :

$$
\chi=\chi_{o}+R M \lambda^{2} \quad R M=\frac{e^{3}}{8 \pi^{2} \epsilon_{o} m_{e}^{2} c^{3}} \int n_{e} \mathbf{B} \cdot d \mathbf{l}
$$

where $\chi$ is the observed polarization angle, $\chi_{0}$ is the intrinsic emitted polarization angle, and the integral is carried out over the line of sight from the source to the observer. Due to the inverse dependence on the square of the mass of the Faraday-rotating particles, it is usually assumed that observed Faraday rotation is due to the action of electrons; because the effective masses of relativistic electrons are higher than those of thermal electrons, these electrons are usually assumed to be thermal. The magnitude of the rotation measure RM depends on both $n_{e}$ and the line-of-sight component of the magnetic field, while the sign of the Faraday rotation is determined by the direction of the line-of-sight magnetic field (either toward or away from the observer). The action of Faraday rotation can be identified through the $\lambda^{2}$ dependence of the observed polarization angle $\chi$.

Note that it is the line-of-sight component of the ambient magnetic field that determines the magnitude and sign of the Faraday rotation. Essentially all observations of extragalactic sources are affected by Faraday rotation to some extent, because their radiation must always pass through our own galaxy on its way to the Earth. Usually, these rotations are not very large at wavelengths of about $6 \mathrm{~cm}$ and shorter.

As was pointed out by Blandford [2], if a jet has a helical B field, we should observe a Faraday-rotation gradient across the jet, due to the systematically changing line-of-sight component of the $\mathbf{B}$ field across the jet. Thus, the detection of transverse Faraday-rotation gradients across AGN jets could potentially provide a powerful diagnostic for the presence of an azimuthal field component associated with a helical or toroidal jet $\mathbf{B}$ field. The theoretical simulations of Broderick and McKinney [3] directly demonstrate the development of helical jet $\mathbf{B}$ fields and the resulting presence of Faraday rotation gradients across the simulated jets.

A number of transverse Faraday-rotation gradients across parsec-scale AGN jets have been reported in the literature; for example, in [4-7]. The largest transverse RM gradient observed to date is that reported in [8] for 3C273, based on their analysis of $43 \mathrm{GHz}$ and $86 \mathrm{GHz}$ Very Long Baseline Array (VLBA) polarization data. A recently detected example is shown in Figure 1 [9]; it is striking that the 
transverse RM gradient is visible across the entire observed core and jet structure. Gómez et al. [10] superposed degree of polarization and RM images for 3C120 obtained at multiple epochs to effectively map out these quantities along a much longer portion of the jet than was possible at any single epoch, making the transverse structure in the polarization and Faraday rotation appreciably clearer.
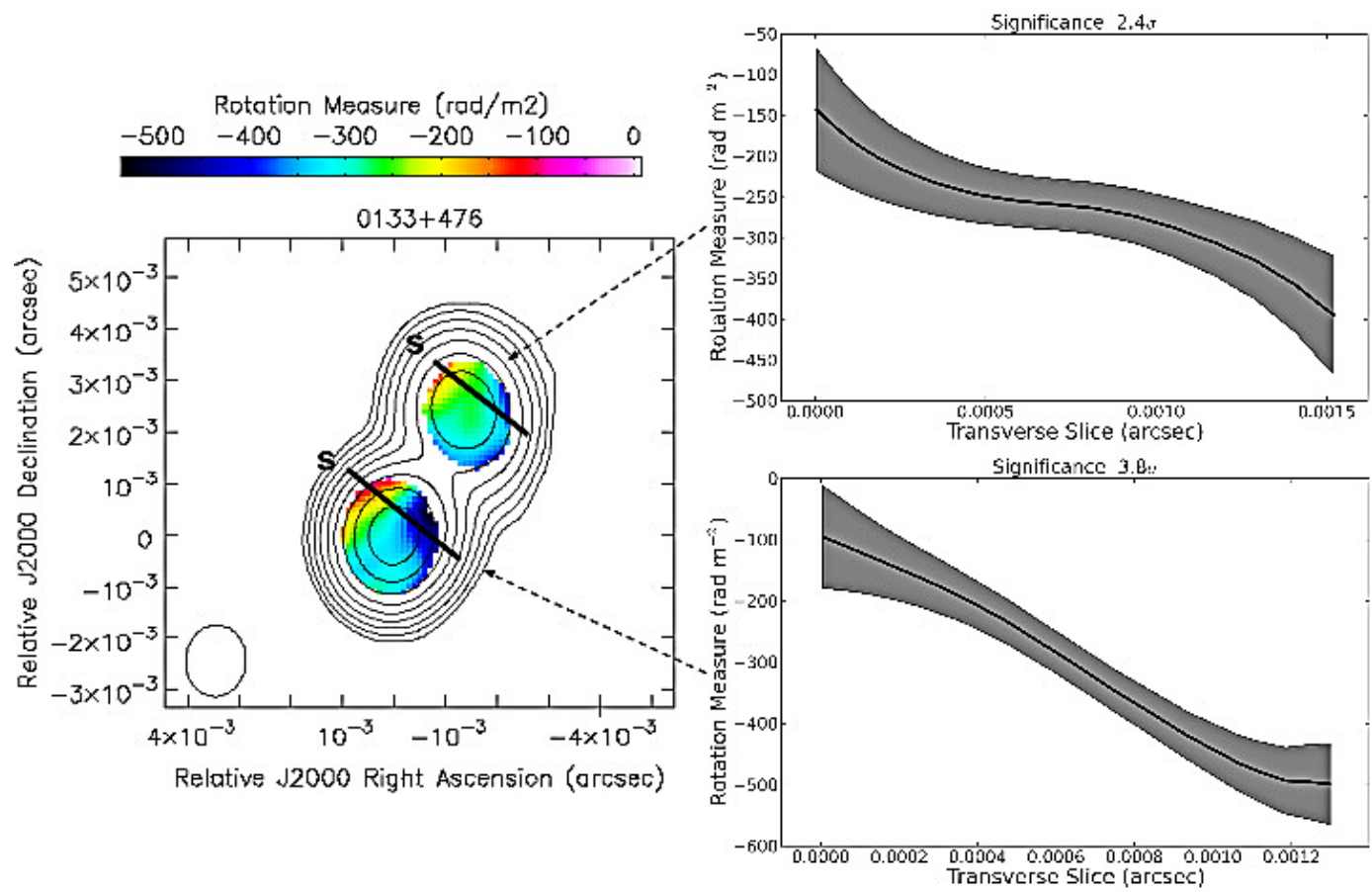

Figure 1. Transverse rotation measure $\left(\mathrm{RM}\right.$, in units of $\left.\mathrm{rad} / \mathrm{m}^{2}\right)$ gradient across the VLBA core region and jet of the Active Galactic Nuclei (AGN) $0133+476$, based on the 8.1-15.3 GHz data of [4], reanalyzed in [9]. Intensity contours with the RM distribution superposed in colour are shown. The beam size is shown in the lower left-hand corner of the map. The panels to the right show RM slices for the regions indicated in the map, with the slices starting with the letters "S" on the left-hand panel. The significances of the two slices are $3.0 \sigma$ (core region) and $2.4 \sigma$ (jet).

Of course, it is also possible to imagine situations when a gradient in the Faraday rotation roughly across a VLBI jet occurs not due to a systematic variation in the line-of-sight $\mathbf{B}$ field component, but due to a gradient in the density of thermal electrons in the region surrounding the jet. This could be the case, for example, if the jet were propagating through a non-uniform medium that was denser on one side of the jet than on the other. In this regard, it is important to point out a crucial discriminator: while transverse RM gradients with RM values of a single sign that increase toward one edge of the jet could potentially be caused by either a helical jet $\mathbf{B}$ field or a density gradient in the surrounding medium, transverse RM gradients that display one sign at one edge of the jet, pass through zero, and then display the other sign at the other edge of the jet are a clear and unambiguous sign of a helical jet $\mathbf{B}$ field. It is therefore highly significant that transverse RM gradients displaying both signs have been observed for a number of AGN, after subtracting the effect of the RM arising in our Galaxy (e.g., [5-7]; in such cases, the only plausible explanation for the observed transverse RM gradients is that the corresponding jets have helical $\mathbf{B}$ fields. Note also that the lack of a sign change in the RM values along a transverse gradient does not rule out the possibility that the gradient is due to a toroidal field component, since some combinations of pitch angle and viewing angle will give transverse gradients of a single sign; in addition, there may be significant Faraday rotation occurring in the AGN host galaxy, which we cannot estimate and remove.

As Sikora et al. [11] have pointed out, the lack of deviations from a $\lambda^{2}$ wavelength dependence for the observed polarization angles in some cases indicates that the Faraday rotation must be external, 
suggesting that the observed Faraday rotation is occurring predominantly in outer layers of the jet, or in the immediate vicinity of the jet. This suggests a picture in which the helical field is present in the jet and its immediate vicinity, but the thermal electrons required for the Faraday rotation are confined to the outer parts of the jet and its vicinity.

In some theoretical pictures (e.g., [12]), a helical magnetic field is generated in the innermost jets, but is disrupted in the core region by either hydrodynamical turbulence-possibly generated by shocks - or magnetohydrodynamical (MHD) turbulence generated by current-driven instabilities. However, the common detection of transverse RM gradients shows that a helical field component can survive to scales well outside the VLBI core. Further, modeling by Marscher [13] has indicated that the disordered field component is able to explain rapid variability in the presence of an appreciable helical field component. This suggests a picture with both helical/toroidal (ordered) and chaotic (turbulent) field components, with the former required to explain the observed transverse RM gradients and the latter required to explain the observed rapid variability, as is shown schematically in Figure 2.

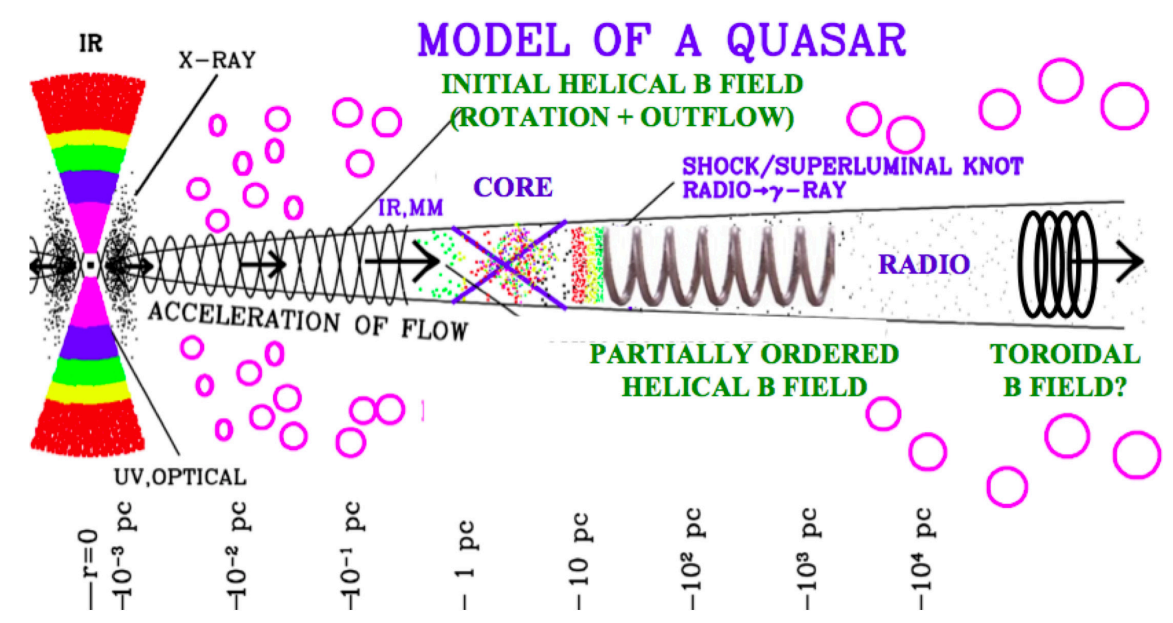

Figure 2. Modification of the well-known schematic of Marscher (e.g., [14]) to reflect the persistence of a helical magnetic-field component beyond the core region. It is expected that the toroidal component of this helical field should become more dominant with distance from the core, due to the more rapid decay of the longitudinal (poloidal) component.

An important question is whether the azimuthal field components revealed by the detected transverse RM gradients are associated with helical or toroidal B fields. Generally speaking, it is only asymmetry of the intensity and polarization profiles across the jet that can distinguish observationally between a helical field (with an ordered poloidal field component) and a toroidal field (with a disordered poloidal field component). The degree of polarization is determined by the component of the jet $\mathbf{B}$ field in the plane of the sky, while the RM is determined by the $\mathbf{B}$ field along the line of sight. The schematic of a helical $\mathbf{B}$ field depicted in the region marked "partially ordered helical $\mathbf{B}$ field" in Figure 2 shows qualitatively that the field is predominantly along the line of sight on one side of the helix (the upper edge of the schematic helical field in the figure), giving rise to higher Faraday rotation and lower degrees of polarization, and predominantly in the plane of the sky at the other side of the helix (the lower edge of the schematic helical field in the figure), giving rise to lower Faraday rotation and higher degrees of polarization. In contrast, in the absence of other factors, a toroidal field should give rise to a symmetric polarization profile across the jet. Fitting of the total intensity and polarization profile across the parsec-scale jet of Mrk501 carried out in [15] shows that the appreciably asymmetric polarization profiles of this AGN jet are fit well using a simple helical-field model; in addition, the parsec-scale jet of Mrk501 exhibits a transverse RM gradient $[7,16]$, with the larger-magnitude RM values present on the side of the jet with a lower degree of polarization, as is expected for a helical jet B field. It is also noteworthy that Zamaninasab et al. [17] found evidence for a 
large-scale helical magnetic field component in 3C454.3 through an analysis of transverse intensity, fractional polarization, polarization angle, and RM profiles of the very well resolved jet of this source. Many AGN jets display significantly asymmetric transverse polarization profiles, and further analyses for such jets that also display transverse RM gradients will show how often the expected patterns for transverse RM gradients associated with helical jet $\mathbf{B}$ fields are observed.

\section{Evidence for the "Return" Field}

Mahmud et al. [5,18] and Gabuzda et al. [6] have reported reversals in the directions of observed transverse Faraday rotation gradients, either with distance along the jet (four AGNs) or with time (two AGNs). Gabuzda et al. [9] have detected another four examples, one of which is shown in Figure 3.

These results at first seem puzzling, since the direction of a transverse RM gradient associated with a helical $\mathbf{B}$ field is essentially determined by the direction of rotation of the central black hole and accretion disk and the initial direction of the poloidal field component that is "wound up" by the rotation. It is difficult in this simplest picture to imagine how the direction of the resulting azimuthal field component could change with distance along the jet or with time. However, in a picture with a nested helical field structure-similar to the "magnetic tower" model of [19], but with the direction of the azimuthal field component being different in the inner and outer regions of helical field-such a change in the direction of the net observed RM gradient could be due to a change in dominance from the inner to the outer region of helical field in terms of their overall contribution to the Faraday rotation [15].

One potential concern is that at least one of the observed RM gradients is typically in the core region, which can include regions that can be quite optically thick. However, it has been argued (e.g., [7]) that the observed core polarization is very likely dominated by the contribution of optically thin regions in the innermost jet, so that it is reasonable to interpret monotonic transverse RM gradients in the core region as evidence for a toroidal field component, particularly when the estimated significance appreciably exceeds $3 \sigma$. Another potential issue with transverse RM gradients with significances exceeding $3 \sigma$ across very compact regions is the limited width spanned by the RM gradients. Inspection of the right-hand panel of Figure 30 of [4] shows that those Monte Carlo simulations yielded spurious $3 \sigma$ transverse RM gradients in about $1 \%$ of trials when the width spanned by the RM gradients were 1.5 beamwidths or less. Although the probability of any single detected narrow RM gradient being spurious is low, studies carried with samples such as that of [4], with 149 sources, could yield 1-3 spurious gradients. An analysis for this sample in [9] yielded transverse RM gradients with significances of $3 \sigma$ or more across the core regions of $\simeq 10$ AGNs—clearly too many for all to be spurious, although a small number of these may be.

Thus, the detection of reversals in the directions of transverse RM gradients may provide the first observational evidence for the presence of the return $\mathbf{B}$ field. One theoretical picture that predicts a nested helical-field structure with oppositely directed azimuthal field components in the inner and outer regions of helical field is the "Poynting-Robertson cosmic battery" model of [20,21], which is considered further in the following section, although other plausible systems of currents may also give rise to similar field configurations.

\section{Global Patterns in the Toroidal B Fields and Axial Currents of AGN Jets}

In some sense, the direction of an observed transverse RM gradient associated with a helical jet $\mathbf{B}$ field will be determined by two factors: the direction of the rotation of the central black hole and its accretion disk (clockwise or counter-clockwise projected onto the sky) and the direction of the initial poloidal component of the "seed field" that is wound up by the rotation (inward or outward along the jet). This initially may lead one to expect that the orientation of these transverse RM gradients should be random on the sky, since there is no obvious physical reason for a preference in the direction of rotation of the central black holes or the initial poloidal seed field. 

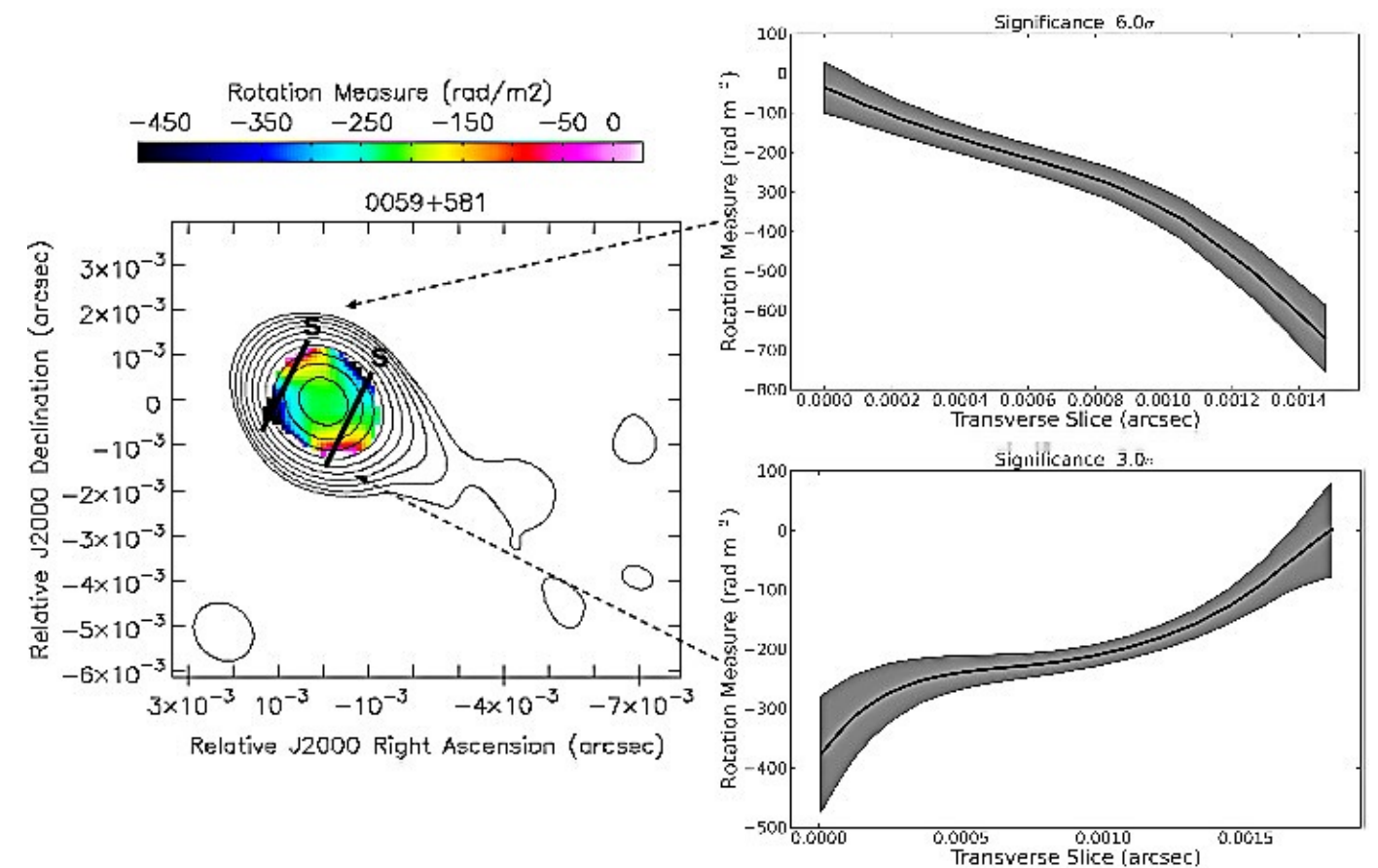

Figure 3. Transverse RM (in units of $\mathrm{rad} / \mathrm{m}^{2}$ ) gradient across the VLBI core region and jet of the AGN $0059+581$, based on the 8.1-15.3 GHz data of [4], reanalyzed in [9]. Intensity contours with the RM distribution superposed in colour are shown. The beam size is shown in the lower left-hand corner of the map. The panels to the right show RM slices for the regions indicated in the map, with the slices starting by the letters "S" on the left-hand panel. The significances of the two slices are $6.0 \sigma$ (core region) and $3.0 \sigma$ (jet), making both of the oppositely directed transverse RM gradients statistically significant.

However, further thought shows that this is not necessarily the case. For example, the presence of an azimuthal jet $\mathbf{B}$ field component implies that the jet carries a current in the region interior to the region where this field component is detected. Let us therefore consider the currents associated with the azimuthal components of helical B fields propagating outward along AGN jets. The simple schematics shown in Figure 4 show that inward/outward jet currents will be associated with characteristic orientations of the azimuthal $\mathbf{B}$ field components, in accordance with the right-hand rule taught in first-year university physics. In turn, these characteristic orientations of the azimuthal field components give rise to characteristic and different orientations of the transverse RM gradients projected onto the sky for inward and outward jet currents. Thus, if the jet currents located interior to the regions where the transverse RM gradients are detected (i.e., closer to the jet axis) have a preferred direction, the orientation of the observed transverse RM gradients will also have a preferred direction, as is illustrated in Figure 4.

This means that any transverse RM gradient can be described as being directed either clockwise (CW, inward current, Figure 4a,c) or counter-clockwise (CCW, outward current, Figure 4b,d) on the sky, relative to the base of the jet. Contopoulos et al. [20] reported evidence for a significant excess of CW transverse Faraday-rotation gradients for parsec-scale AGN jets, based on transverse Faraday-rotation gradients identified in maps from the literature. A re-analysis of the transverse Faraday-rotation gradients considered in [20] to verify their reliability applying the new error-estimation procedure of [4] has been carried out in [7]; this indicated that only a small number of the RM gradients considered in [20] were not statistically significant. Together with additional new detections of transverse RM gradients reported in [6] and currently being analyzed in [9], the collected results confirm a predominance of CW transverse RM gradients (inward jet currents) on parsec scales, with the probability that this asymmetry came about by chance being no more than $1 \%$. 

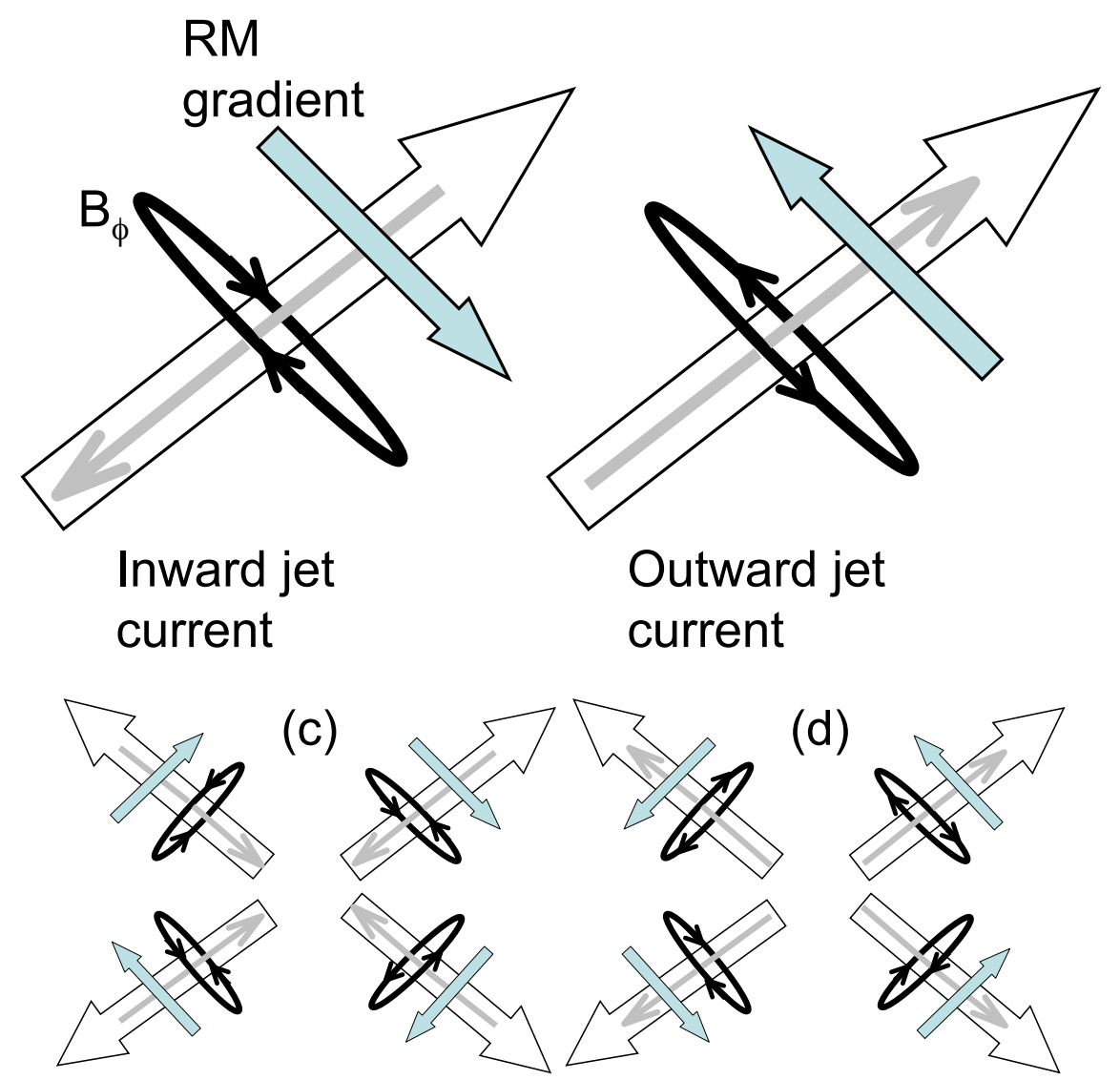

Figure 4. Relationship between the direction of a jet current, its azimuthal $\mathbf{B}$ field $\mathrm{B}_{\phi}$, and the resulting transverse RM gradient for (a) inward; and (b) outward jet currents. In each panel, the large hollow arrow shows the jet direction, the grey arrow the direction of the jet current, the bold circle the direction of $\mathrm{B}_{\phi}$, and the filled arrow crossing the jet the direction of the transverse RM gradient; (c) Same as panel (a) for jets in four different directions; the orientations of the transverse RM gradients form a clockwise pattern on the sky; (d) Same as panel (b) for jets in four different directions; the orientations of the transverse RM gradients form a counter-clockwise pattern on the sky.

This result thus implies a predominance of inward jet currents on parsec scales. Contopoulos et al. [20] suggest that this can be explained via the action of a mechanism they call the "Poynting-Robertson cosmic battery", in which the direction of the disc rotation and the direction of the poloidal field that is "wound up" are coupled in such a way that the resulting azimuthal B field component corresponds to an inward current along the jet axis, independent of the direction of the disc rotation as seen by the observer. This battery mechanism should give rise to a nested-helical-field picture such as has been used to explain reversals in the direction of the observed RM gradients with distance from the core in a number of sources: the inner/outer regions of helical field are associated with inward/outward currents, which give rise to CW/CCW transverse RM gradients.

This leads to the prediction that the inner region of helical field- surrounding a region of inward current-should dominate on parsec scales, while the outer region of helical field—surrounding a region of net outward current-should dominate on appreciably larger scales. Qualitatively speaking, these currents should close in the accretion disk and far out in the kiloparsec radio lobes, forming a self-consistent system of currents and associated helical/toroidal fields. This picture is supported by the analysis of Christodoulou et al. [21], who considered all known detections of transverse RM gradients on scales corresponding to projected distances greater than about $20 \mathrm{pc}$ from the jet base. 
They found strong evidence for a predominance of CCW transverse RM gradients (outward currents) on these scales. This is consistent with the schematic picture shown in Figure 5. It is reasonable to expect that the inward current near the jet axis decreases with distance from the jet base, so that the inner region of helical field associated with this current is more dominant on smaller scales. Christodoulou et al. [21] have also pointed out that $\mathbf{B}$ fields close to the jet axis will acquire a substantial toroidal component closer to the jet base than will $\mathbf{B}$ fields anchored farther out in the accretion disk, making the contribution of the outer region of the helical field increase with distance from the jet base. Together with an enhancement in the amount of thermal material in regions relatively far from the jet axis on kiloparsec scales, this apparently leads to an overall dominance of these outer regions in the observed Faraday rotation on larger scales.

Whether or not their origin is the battery mechanism promoted by [20,21], the collected data for transverse RM gradients across AGN jets on parsec to kiloparsec scales points toward a global pattern of fields and currents, with an inward current near the jet axis and an outward current farther from the jet axis, with an associated nested helical field structure.

\section{Conclusions}

It is widely expected theoretically that a helical jet B field component should form due to the combination of the rotation of the central black hole and its accretion disk and the jet outflow; this has also been demonstrated by computational simulations, such as those of [3]. Because Faraday rotation measure is sensitive to the line-of-sight component of the magnetic field in the region of Faraday rotation, the presence of an azimuthal (toroidal) jet $\mathbf{B}$ field component will give rise to a monotonic gradient of the RM across the jet. Of course, there may also be a random component of the RM associated with turbulent or chaotic motions of material in the vicinity of the jet, which would tend to "blur" this pattern, and the signal-to-noise of the RM measurements may not always be sufficiently high to reliably display the RM gradient, even if present. Nevertheless, statistically significant transverse RM gradients providing direct evidence for helical/toroidal jet $\mathbf{B}$ fields have now been detected in nearly 40 AGN on parsec scales. This demonstrates that a helical field component survives to parsec scales, to distances well beyond the VLBI core.

Reversals of the direction of the transverse RM gradients with distance from the core have now been observed in six AGN [5,6,9]. This provides observational evidence for a "return B field" that forms a nested helical $\mathbf{B}$ field structure with the inner and outer regions of helical field having oppositely directed azimuthal field components.

Statistically significant transverse RM gradients have now also been reliably detected on scales of several tens to thousands of parsecs in 14 AGN, with tentative transverse RM gradients (whose statistical significances do not reach $3 \sigma$ or have not yet been determined) observed on such scales in another six AGN [21-23]. The collected data for transverse RM gradients observed on parsec to kiloparsec scales clearly indicates a predominance of inward currents near the jet axis on parsec scales and outward currents flowing along the jet farther from the axis on scales greater than a few tens of parsec. It is noteworthy that in five of the six cases of reversals of the transverse RM gradients with distance from the core detected so far, the observed RM gradients closer to the jet base are CW and the $\mathrm{RM}$ gradients farther from the jet base CCW on the sky, consistent with this picture. The probabilities that the predominance of CW/CCW RM gradients on parsec/decaparsec-kiloparsec scales came about by chance are both separately no more than $1 \%$; the joint probability that this overall pattern has come about by chance is accordingly much less than $1 \%$, and needs to be taken seriously when considering the overall $\mathbf{B}$ field structures of AGN jets. 


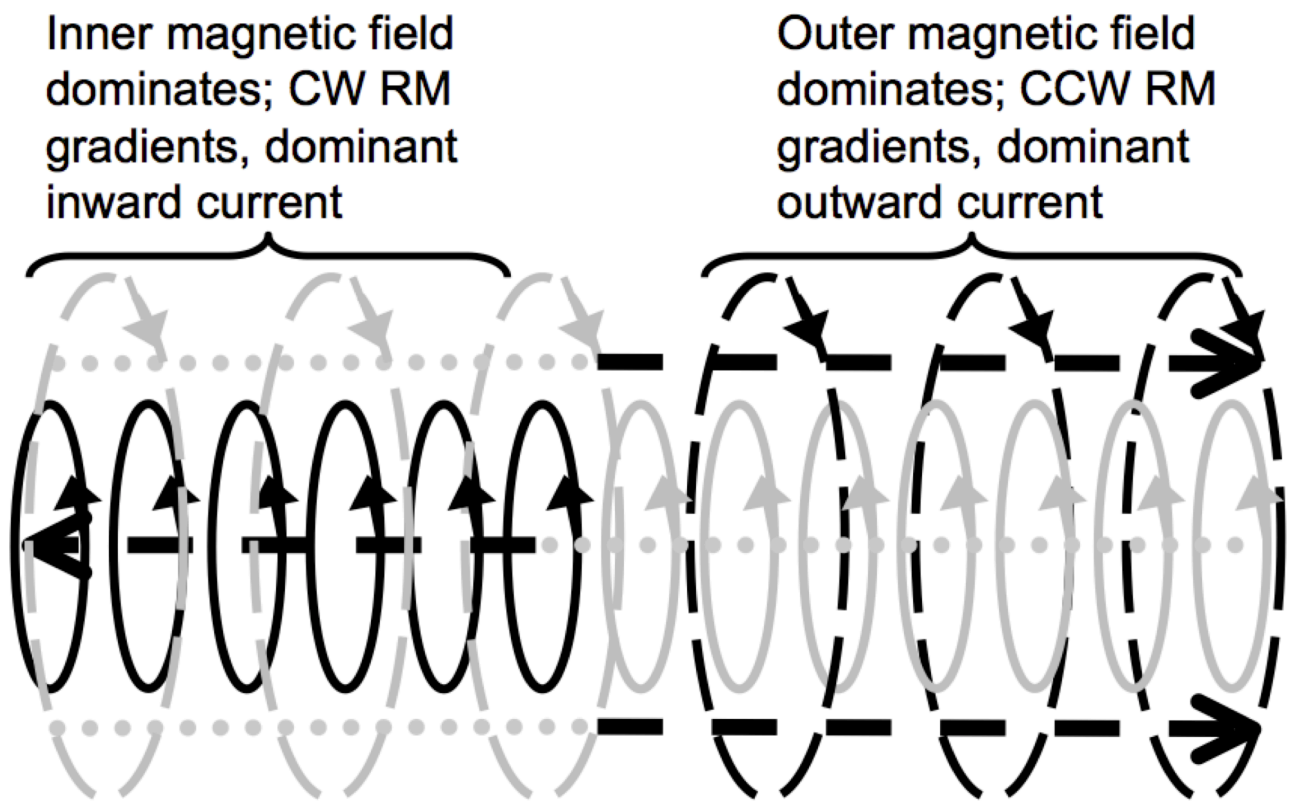

Figure 5. Schematic of a nested helical B field structure with its associated system of currents, with an inward current along the jet axis and an outward current further from the jet axis, associated with the "return" field. The jet propagates to the right, and the jet base is located to the left of the regions shown. The ellipses represent the azimuthal components of the fields; these are shown black and grey at distances where the inner and outer azimuthal fields dominate or do not dominate the observed transverse RM gradients. The orientations of the azimuthal fields are shown by arrows. The associated currents are shown by black dashed and grey dotted lines with arrows. Inward currents dominate on parsec scales, while outward currents dominate on decaparsec-kiloparsec scales. CW: clockwise; CCW: counter-clockwise.

In summary, putting these various pieces of observational information together, the data for transverse RM gradients across AGN jets (probing the azimuthal component of the jet $\mathbf{B}$ field) on parsec to kiloparsec scales points toward a global pattern of fields and currents, with an inward current near the jet axis and an outward current farther from the jet axis. The axial inward jet current is surrounded by a region of helical $\mathbf{B}$ field that gives rise to clockwise RM gradients projected onto the sky, and the outward current farther from the jet axis is surrounded by another region of helical $\mathbf{B}$ field that gives rise to counter-clockwise RM gradients projected onto the sky. Together, these form a nested helical field structure, with this associated system of currents, which close in the accretion disk and far out in the kiloparsec-scale radio lobes. This provides absolutely fundamental information about the jets as electromagnetic structures, and theoretical modeling aimed at identifying the most physically likely mechanism giving rise to this global field-current structure will be extremely important for our understanding of AGN jets.

Acknowledgments: Thanks to Naomi Roche and Matt Nagle for their assistance in making some of the images presented in this paper.

Conflicts of Interest: The author declares no conflict of interest.

\section{References}

1. Kellermann, K.I.; Sramek R.; Schmidt, M.; Shaffer, D.B.; Green, R. VLA observations of objects in the Palomar Bright Quasar Survey. Astron. J. 1989, 98, 1195-1207.

2. Blandford, R.D. Astrophysical Jets; Cambridge University Press: Cambridge, UK, 1993; p. 15.

3. Broderick, A.E.; McKinney, J.C. Parsec-scale Faraday Rotation Measures from General Relativistic Magnetohydrodynamic Simulations of Active Galactic Nucleus Jets. Astrophys. J. 2010, 725, 750-773. 
4. Hovatta, T.; Lister, M.L.; Aller, M.F.; Aller, H.D.; Homan, D.C.; Kovalev, Y.Y.; Pushkarev, A.B.; Savolainen, T. MOJAVE: Monitoring of Jets in Active Galactic Nuclei with VLBA Experiments. VIII. Faraday Rotation in Parsec-scale AGN Jets. Astron. J. 2012, 144, 105-138.

5. Mahmud, M.; Coughlan, C.P.; Murphy, E.; Gabuzda, D.C. Connecting magnetic towers with Faraday rotation gradients in active galactic nuclei jets. Mon. Not. R. Astron. Soc. 2013, 431, 695-709.

6. Gabuzda, D.C.; Reichstein, A.R.; O'Neill, E.L. Are spine-sheath polarization structures in the jets of active galactic nuclei associated with helical magnetic fields? Mon. Not. R. Astron. Soc. 2014, 444, 172-184.

7. Gabuzda, D.C.; Knuettel, S.; Reardon, B. Transverse Faraday-rotation gradients across the jets of 15 active galactic nuclei. Mon. Not. R. Astron. Soc. 2015, 450, 2441-2450.

8. Attridge, J.M.; Wardle, J.F.C.; Homan, D.C. Concurrent 43 and 86 GHz Very Long Baseline Polarimetry of 3C 273. Astrophys. J. Lett. 2005, 633, L85-L88.

9. Gabuzda, D.C.; Nagle, M.; Roche, N. The global pattern of the magnetic fields and currents associated with the jets of active galactic nuclei. Mon. Not. R. Astron. Soc. 2017, in preparation.

10. Gómez, J.L.; Marscher, A.P.; Jorstad, S.G.; Agudo, I.; Roca-Sogorb, M. On the Source of Faraday Rotation in the Jet of the Radio Galaxy 3C120. Astrophys. J. 2011, 733, 11-22.

11. Sikora, M.; Begelman, M.C.; Madejski, G.M.; Lasota, J.-P. Are Quasar Jets Dominated by Poynting Flux? Astrophys. J. 2005, 625, 72-77.

12. Marscher, A.P. Turbulent, Extreme Multi-zone Model for Simulating Flux and Polarization Variability in Blazars. Astrophys. J. 2014, 780, 87-96.

13. Marscher, A.P. Time-variable linear polarization as a probe of the physical conditions in the compact jets of blazars. In Extragalactic Jets from Every Angle; Massaro, F., Cheung, C.C., Lopez, E., Siemiginowska, A., Eds.; Cambridge University Press: Cambridge, UK, 2015; pp. 122-127.

14. Research of the Blazar Group, Boston University Institute for Astrophysical Research. Available online: https://www.bu.edu/blazars/research.html (accessed on 30 January 2017).

15. Murphy, E.; Cawthorne, T.V.; Gabuzda, D.C. Analysing the transverse structure of the relativistic jets of active galactic nuclei. Mon. Not. R. Astron. Soc. 2013, 430, 1504-1505.

16. Croke, S.M.; O'Sullivan, S.P.; Gabuzda, D.C. The parsec-scale distributions of intensity, linear polarization and Faraday rotation in the core and jet of Mrk501 at 8.4-1.6 GHz. Mon. Not. R. Astron. Soc. 2010, 402, 259-270.

17. Zamaninasab, M.; Savolainen, T.; Clausen-Brown, E.; Hovatta, T.; Lister, M.L.; Krichbaum, T.P.; Kovalev, Y.Y.; Pushkarev, A.B. Evidence for a large-scale helical magnetic field in the quasar 3C 454.3. Mon. Not. R. Astron. Soc. 2013, 436, 3341-3356.

18. Mahmud, M.; Gabuzda, D.C.; Bezrukovs, V. Surprising evolution of the parsec-scale Faraday Rotation gradients in the jet of the BL Lac object B1803 + 784. Mon. Not. R. Astron. Soc. 2009, 400, 2-12.

19. Lynden-Bell, D.B. Magnetic collimation by accretion discs of quasars and stars. Mon. Not. R. Astron. Soc. 1996, 279, 389-401.

20. Contopoulos, I.; Christodoulou, D.M.; Kazanas, D.; Gabuzda, D.C. The Invariant Twist of Magnetic Fields in the Relativistic Jets of Active Galactic Nuclei. Astrophys. J. Lett. 2009, 702, L148-L152.

21. Christodoulou, D.M.; Gabuzda, D.C.; Knuettel, S.; Contopoulos, I.; Kazanas, D.; Coughlan, C.P. Dominance of outflowing electric currents on decaparsec to kiloparsec scales in extragalactic jets. Astron. Astrophys. 2016, 591, A61-A71.

22. Motter, J.; Gabuzda, D.C. 18-22 cm VLBA Observational Evidence for Toroidal B-Field Components in Six AGN Jets. Galaxies 2016, 4, 18.

23. Motter, J.; Gabuzda, D.C. 18-22 cm VLBA Faraday rotation studies for six AGN jets. Mon. Not. R. Astron. Soc. 2017, in press.

(C) 2017 by the author; licensee MDPI, Basel, Switzerland. This article is an open access article distributed under the terms and conditions of the Creative Commons Attribution (CC-BY) license (http://creativecommons.org/licenses/by/4.0/). 